\title{
Functional Group Interconversion of Alkylidenemalononitriles to Primary Alcohols by a Cooperative Redox Operation
}

\author{
Fabien Emmetiere \\ Alexander J. Grenning* (D) \\ Department of Chemistry, University of Florida, \\ PO Box 117200, Gainesville, FL 32611-7200, USA \\ grenning@ufl.edu
}

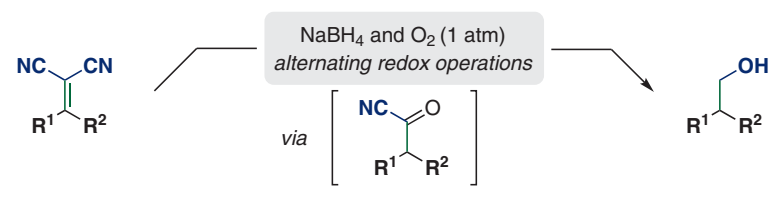

would be generally valuable to chemical synthesis and (2) the transformation would require a cooperative redox operation: Could a reductant and an oxidant cohabitate a reaction medium to achieve an alternating reduction/oxidation/reduction sequence without neutralizing themselves? If conditions and compatible reductant and oxidant could be identified, then alkylidenemalononitriles could be directly converted into $1^{\circ}$ alcohols (Scheme $1 \mathrm{~B}$ ). While there are obvious challenges to redox transformations of this type, there are known and effective combinations of reductant/oxidant for chemical transformations. ${ }^{22-27}$ For example, molecular oxygen is compatible with mild reductants such as borohydrides $\left(\mathrm{NaBH}_{4}, \mathrm{Bu}_{4} \mathrm{NBH}_{4}\right)^{25,28-33}$ and silanes $\left(\mathrm{Et}_{3} \mathrm{SiH} \text { and } \mathrm{PhSiH}_{3}\right)^{34-38}$ and these redox partners have been utilized to achieve a variety of transformations, such as aerobic oxidation of alkenes or hydrogenation reactions. ${ }^{39,40}$ In terms of other oxidants, tert-butyl hydroperoxide has been used in combination with $\mathrm{PhSiH}_{3}$ to achieve alkene reductions via metal-catalyzed hydrogen atom transfer (HAT). ${ }^{41-}$ ${ }^{44}$ As a final example, Toste and co-workers have shown that phase separation of oxopiperidinium (oxidant; aqueous phase) and Hantzsch ester (reductant; solid phase) could allow for controlled redox transformation. ${ }^{45}$ Inspired by this

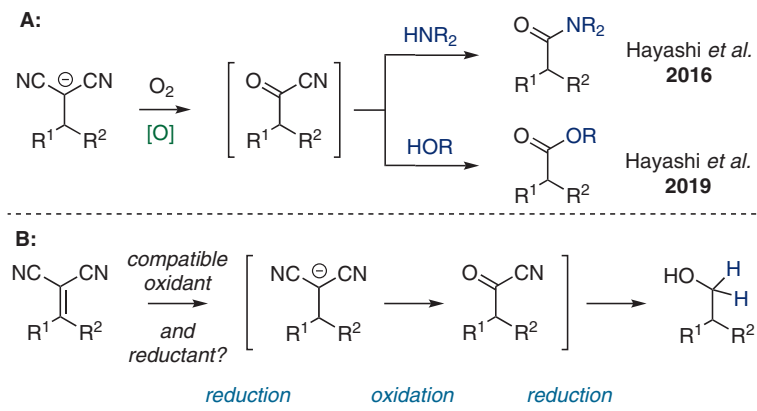

Scheme 1 A: Relevant oxidative decyanation strategies. ${ }^{19,20}$ B: This report: alkylidenemalononitrile functional group interconversion to 1 alcohol by a redox transformation. 
literature precedent, we now report that the combination of oxygen and $\mathrm{NaBH}_{4}$ can interconvert alkylidenemalononitriles to $1^{\circ}$ alcohols by an alternating reduction/oxidation/reduction sequence.

To begin our studies, we optimized the proposed redox functional group interconversion as described in Table 1. Utilizing model substrate 1, our first success was realized when the transformation was performed in polar solvents with oxygen ( $1 \mathrm{~atm}$ ) as the oxidant and $\mathrm{NaBH}_{4}$ as the reductant (entries 2-4). Choice of solvent was crucial as we observed significant amounts of methyl ester byproduct in methanol ${ }^{19,20}$ and no conversion in nonpolar solvents due to insolubility of the $\mathrm{NaBH}_{4}$, presumably (entry 1). As previously reported by Hayashi and co-workers, ${ }^{19,20}$ the reaction had to be performed under strictly anhydrous conditions to prevent the formation of carboxylic acid byproducts. The use of molecular sieves was therefore imperative to maximize conversion into the desired product. Addition of potassium carbonate in the reaction mixture favored the formation of desired product 3a (entries 5, 6). We speculate that the addition of a base in the system results in a higher concentration of alkylmalononitrile anion, which the transformation must progress through. Finally, by adjusting the reaction time and temperature to 1.5 hours and $50{ }^{\circ} \mathrm{C}$ respectively, we were able to form $3 a$ as a sole product during this transformation (entry 8). Interestingly, the reaction could be conducted under a constant flow of air (entry 9), though the transformation was significantly slower. It should also be noted that the conversion from 1 into 2 and/or 3a was excellent in all cases. Indeed, reduction of the alkylidenemalononitrile was extremely facile (completed within minutes, observable by TLC). We found that the ratedetermining step was the oxidation from $\mathbf{2}$ to $3 \mathbf{a}$ which was considerably slower.

\begin{tabular}{|c|c|c|c|c|c|c|}
\hline \multicolumn{2}{|c|}{$\mathrm{Ph}_{1}^{\mathrm{NC}}$} & \multicolumn{2}{|c|}{$\begin{array}{c}\mathrm{NaBH}_{4} \text { (4 equiv.) } \\
\text { Base, } \mathrm{O}_{2} \text { (2 equiv.) }\end{array}$} & \multicolumn{2}{|c|}{${ }^{N C}$} & \multirow{2}{*}{$\begin{array}{l}\text { \% Conversion } \\
\begin{array}{l}\text { (NMR) } \\
\text { (NMh }\end{array}\end{array}$} \\
\hline Entry & $\begin{array}{l}\text { Base } \\
\text { ( } 2 \text { equiv.) }\end{array}$ & $\begin{array}{l}\text { Solvent } \\
(0.1 \mathrm{M})\end{array}$ & $\begin{array}{l}\text { Temp } \\
\left({ }^{\circ} \mathrm{C}\right)\end{array}$ & $\begin{array}{l}\text { Time } \\
\text { (h) }\end{array}$ & $\begin{array}{l}\text { Ratio } \\
2 / 3 a\end{array}$ & \\
\hline 1 & - & toluene & $\mathrm{rt}$ & 1 & $0: 0$ & 0 \\
\hline 2 & - & DMSO & $\mathrm{rt}$ & 1 & $50: 50$ & 95 \\
\hline 3 & - & $\mathrm{MeCN}$ & it & 1 & $47: 53$ & 97 \\
\hline 4 & - & DMF & $\mathrm{rt}$ & 1 & $33: 66$ & 96 \\
\hline 5 & $\mathrm{~K}_{2} \mathrm{CO}_{3}$ & $\mathrm{MeCN}$ & rt & 1 & $40: 60$ & 98 \\
\hline 6 & $\mathrm{~K}_{2} \mathrm{CO}_{3}$ & DMF & $\mathrm{rt}$ & 1 & $30: 70$ & $>99$ \\
\hline 7 & $\mathrm{~K}_{2} \mathrm{CO}_{3}$ & DMF & rt & 4.5 & $17: 83$ & $>99$ \\
\hline 8 & $\mathrm{~K}_{2} \mathrm{CO}_{3}$ & DMF & 50 & 1.5 & $0: 100$ & $>99$ \\
\hline $9^{a}$ & $\mathrm{~K}_{2} \mathrm{CO}_{3}$ & DMF & 50 & 12 & $73: 27$ & $>99$ \\
\hline
\end{tabular}

${ }^{\text {a }}$ Reaction performed under air instead of $\mathrm{O}_{2}$.
Having found the optimized conditions, we next explored the scope utilizing a variety of alkylidenemalononitriles (Scheme 2). The initial model substrate 1 was converted into its respective $1^{\circ}$ alcohol $3 a$ in $62 \%$ yield. A variety of other arene-containing products $\mathbf{3 b} \mathbf{b} \mathbf{3 d}$ were successfully prepared. As the transformation was optimized for a mild reductant, $\mathrm{NaBH}_{4}$, chemoselective transformation of the alkylidenemalononitrile can be achieved over other reducible functional groups such as esters, alkenes, and carbamates (e.g., 3e-3g, respectively). Considering this, we prepared an $\alpha$-ester-functionalized alkylidenemalononitrile which can undergo the desired functional group interconversion to the $1^{\circ}$ alcohol with concomitant lactonization to $3 \mathrm{~m}$ in $65 \%$ yield. Similarly, oxygen is a fairly gentle oxidant. We found that $\mathrm{e}^{-}$-rich arenes ( $\mathbf{3 h}$ ), and to a less extent pyridines (3i), could be tolerated in the transformation. We also uncovered some functional groups that were only modestly tolerated including allenes, phenols, alkylamines, and alkynes (3j-31, 3n, respectively). We suspect that these compounds are sensitive to the $\mathrm{NaBH}_{4} / \mathrm{O}_{2}$ conditions. An increased tolerance to these functional groups will be investigated in the future.

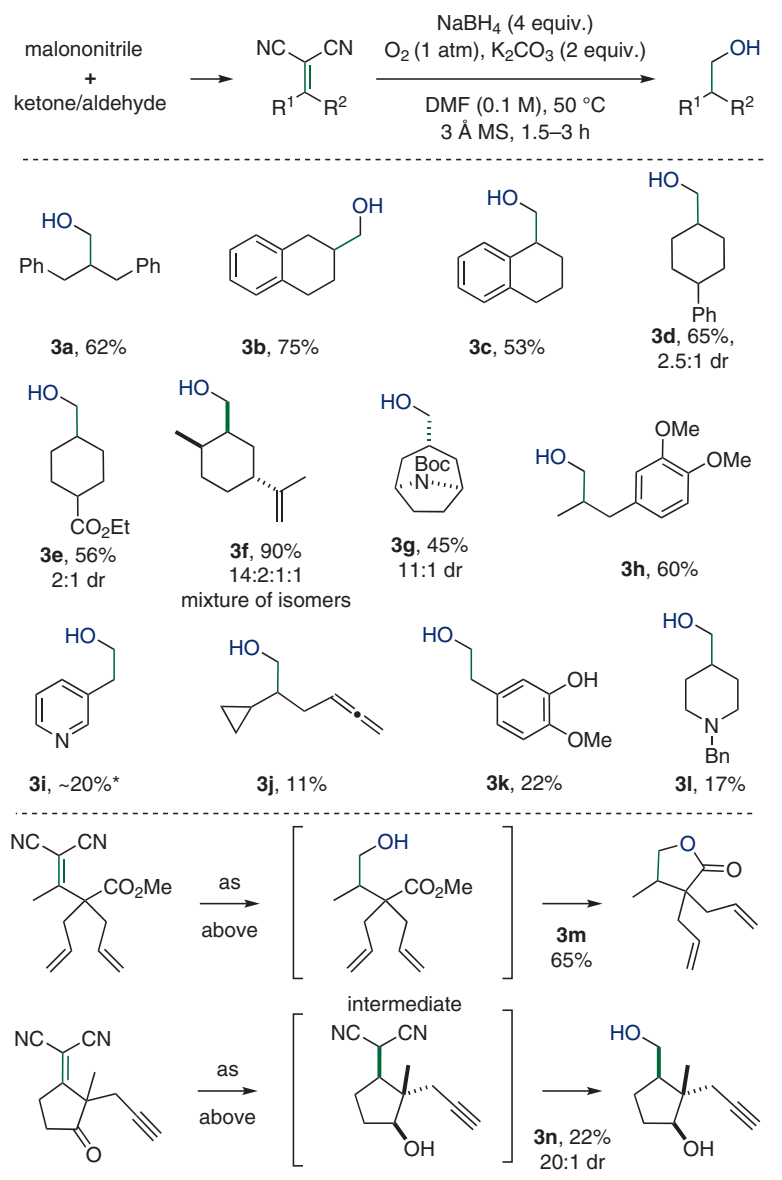

* $3 \mathbf{i}$ was obtained as an impure mixture (see NMR spectra for more details)

Scheme 2 Reaction scope 
Based on our scope from Scheme 2 and a comparison of literature strategies to prepare some of these molecules, it became apparent that this sequence (Knoevenagel condensation and redox functional group interconversion) is an attractive alternative to the known methods. For example, it is common to take a commercial ketone and yield the targeted alcohol $\mathbf{3 g}$ by Wittig olefination and $\mathrm{BH}_{3}$ reduction (Scheme 3). ${ }^{46,47}$ Knoevenagel condensation and $\mathrm{O}_{2} / \mathrm{NaBH}_{4}$ functional group interconversion have the potential to be a more practical/operationally simple two-step sequence as it avoids pyrophoric and air-sensitive butyllithium and boranes.

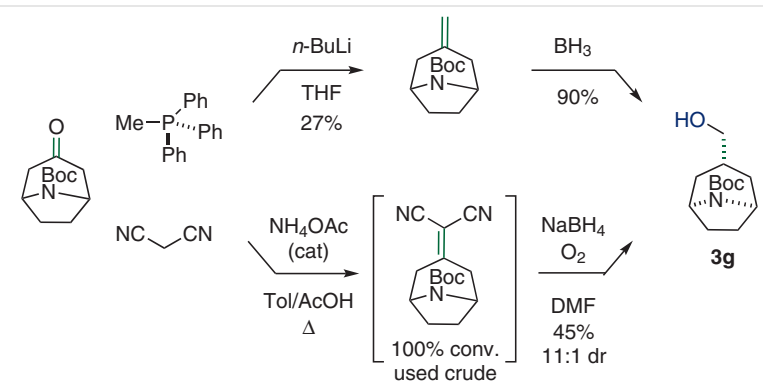

Scheme 3 Comparison of a common literature route to this report's method for converting a ketone into a homologated alcohol

Having a better understanding of the substrate scope and limitations for alkylidenemalononitrile redox functional group interconversion to $1^{\circ}$ alcohols, we wished to apply this transformation to $\gamma$-allyl-alkylidenemalononitriles (Scheme 4A and Scheme 5). Such scaffolds would be readily available from alkylidenemalononitriles and allylic electrophiles by deconjugative allylation yielding 3,3-dicyano-1,5dienes followed by thermal Cope rearrangement. ${ }^{21,48-53}$ Thus alkylidenemalononitrile redox functional group interconversion to $1^{\circ}$ alcohols would provide a straightforward route to 1,6-hexenols $\mathbf{4 a - 4 d}$. Notably, such products would bear resemblance to 'oxy-Cope products', ${ }^{4-57}$ but via an alternative route (Scheme 4A vs Scheme 4B). To examine this, we prepared several $\gamma$-allyl-alkylidenemalononitriles or reduced variants. Regarding the reduced variants, in some of our previous work, we developed 'reductive Cope rearrangements' to promote thermodynamically favorable Cope rearrangements. ${ }^{49,53} \mathbf{2 b - 2 d}$ (Scheme 5) were prepared as such. Regarding the redox functional group interconversion to $1^{\circ}$ alcohols, the reaction was successful at yielding a variety of 1,6-hexenols with unique substitution patterns and functional groups. In this regard, 4a bears a ketal and a 1,3diphenylallyl moiety, $\mathbf{4 b}$ is monoterpene derived (myrtenol), and 4d has an additional ester functional group.

1,6-Hexenols, such as those prepared in Scheme 5, are polyfunctional and can be utilized in chemical synthesis to make valuable scaffolds. As final aspect of this study, we aimed to demonstrate that terpenoid-like scaffolds can be accessed by straightforward sequences (Scheme 6). Starting
A: Synthesis of 1,6-hexenols by Cope rearrangement and redox functional group interconversion

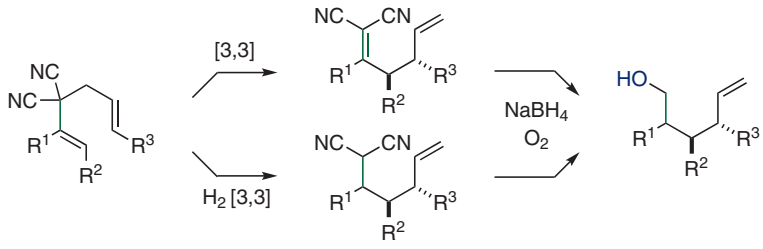

B:The above sequence yields similar products to the oxy-Cope rearrangement

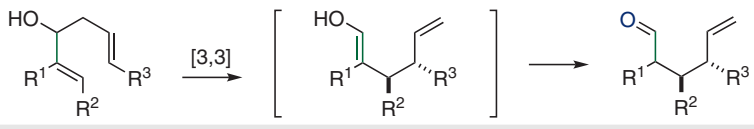

Scheme 4 Routes to building blocks with 1,5-enols bicyclic ring system 5a in four steps: (i) alcohol oxidation, (ii) Brown allylation, (iii) ring-closing metathesis, and (iv) alcohol oxidation. Notably, multiple consecutive steps could be telescoped reducing the need of intermediate purifications (see the Supporting Information for details). Thus, yields are reported after the final step when chromatographic purification was performed. 5a maps well onto the daphnane and tigliane terpenoid scaffolds, among others, and bears a variety of other functionality for further chemical transformation. A few other related scaffolds 6-9 were prepared by similar telescoped sequences.

In conclusion, we have developed a strategy to convert alkylidenemalononitriles into primary alcohols. The reaction relies on a choreographed redox process involving alkylidene reduction, malononitrile oxidation, and acylcyanide reduction where molecular oxygen and $\mathrm{NaBH}_{4}$ work 


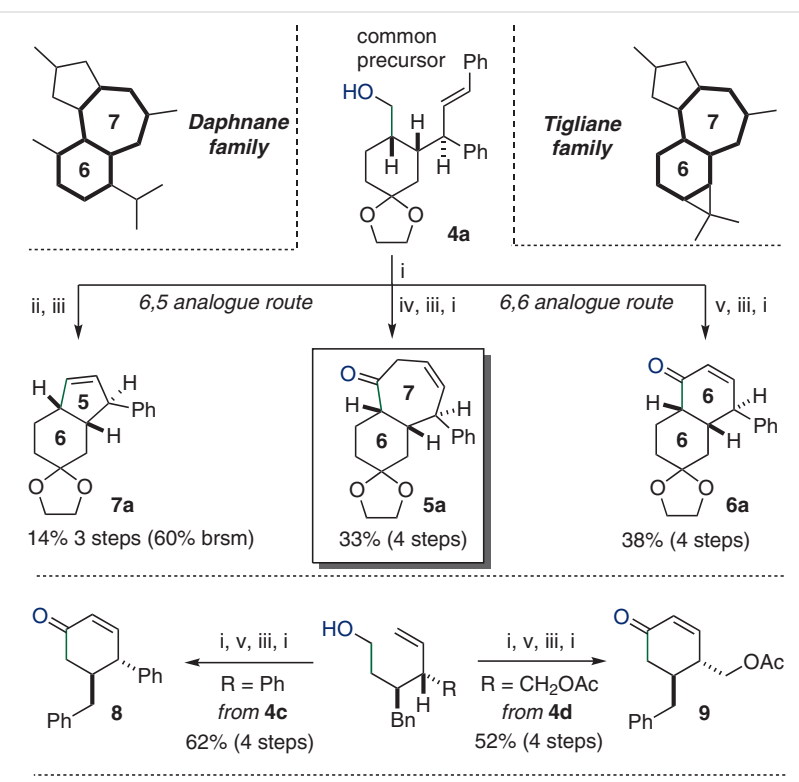

Conditions: i) 1.5 equiv. PCC, DCM, rt. ii) 2 equiv. $\mathrm{MePPh}_{3} \mathrm{Br}, 2.5$ equiv. ${ }^{\mathrm{B}} \mathrm{BuOK}$, THF, $0{ }^{\circ} \mathrm{C}$. iii) $1 \mathrm{~mol} \%$ Grubbs II, toluene, $80^{\circ} \mathrm{C}$. iv) 1.5 equiv. allylboronic acid pinacol ester, toluene, $80^{\circ} \mathrm{C}$. v) 1.3 equiv. vinylmagnesium bromide, THF, $-78^{\circ} \mathrm{C}$.

Scheme 6 Proof-of-concept syntheses toward terpene natural products

cooperatively. The method was applied to a variety of carbon skeletons and was utilized to synthesize complex terpenoid architectures. Future directions will include utilization of the transformation in synthesis and further optimization and scope studies as needed.

All commercial materials were used without further purification. Knoevenagel adducts were synthesized from modified literature procedures that are referenced along the way. All other synthetic protocols are outlined below. ${ }^{1} \mathrm{H}$ NMR and ${ }^{13} \mathrm{C}$ NMR spectra were recorded in $\mathrm{CDCl}_{3}$ using a $400 \mathrm{MHz}$ or $600 \mathrm{MHz}$ Varian VNMRS spectrometer (with $\mathrm{CHCl}_{3}$ residual peak as an internal standard) unless specified otherwise. All ${ }^{13} \mathrm{C}$ NMR spectra were recorded with complete proton decoupling. HRMS data were recorded on an Agilent Time-of-Flight 6200 spectrometer. Reaction progress was monitored by TLC and visualized by UV light (fluorescence observed by eye after excitation under UV light), phosphomolybdic acid stain, and $\mathrm{KMnO}_{4}$ stain. Compounds were purified via silica gel column chromatography using hexanes/ethyl acetate (Hex/EtOAc) solvent mixtures unless specified otherwise. Notation: Hex/EtOAc $10 \%$ reads as 'mixture of $10 \%$ ethyl acetate in hexanes'. All reactions were carried out using anhydrous solvents obtained dried by passing through activated alumina columns. Solvents used for oxidative decyanations were freeze-pumpthawed three times before use.

One-Pot Oxidative Decyanation of Alkylidenemalononitriles to Alcohols; General Procedures A-1 and A-2

The corresponding alkylidenemalononitrile (procedure A-1) or alkylmalononitrile (procedure A-2) substrate (1 equiv.) was dissolved in $\operatorname{DMF}(0.1 \mathrm{M})$ in a flame-dried Schlenk flask under positive pressure $\mathrm{N}_{2}$ along with 3 Å molecular sieves ( $20 \mathrm{mg} / \mathrm{mL}$ of solvent). Using an $\mathrm{O}_{2}$ balloon, $\mathrm{O}_{2}$ gas was gently bubbled though the reaction mixture using a long needle through the reaction flask septum. $\mathrm{O}_{2}$ was bubbled for $10 \mathrm{~min}$. After $10 \mathrm{~min}, \mathrm{NaBH}_{4}$ (4 equiv. for alkylidenemalononitriles and 2 equiv. for alkylmalononitriles) was then added and the reaction mixture was stirred for 2-5 min (until effervescence stopped). Then, $\mathrm{K}_{2} \mathrm{CO}_{3}$ (2 equiv.) was added and the reaction mixture was heated at $50{ }^{\circ} \mathrm{C} . \mathrm{O}_{2}$ was bubbled through the mixture until reaction completion. Once completion was achieved (monitored by TLC), the reaction mixture was cooled to $\mathrm{rt}$ and the $\mathrm{O}_{2}$ balloon was removed. The excess $\mathrm{NaBH}_{4}$ was slowly quenched with $\mathrm{H}_{2} \mathrm{O}(1 \mathrm{~mL})$ and the reaction mixture was diluted with $2 \mathrm{M} \mathrm{HCl}_{(\mathrm{aq})}$ (5 times the reaction volume). The resulting aqueous mixture was extracted with EtOAc three times (2 times the aqueous phase volume). The combined organic layers were washed with $2 \mathrm{M} \mathrm{HCl}_{(\mathrm{aq})}$ (equal volume as the organic phase) and brine (equal volume as the organic phase), and dried over $\mathrm{Na}_{2} \mathrm{SO}_{4}$. The EtOAc was removed under reduced pressure and the crude was purified via silica gel column chromatography (Hex/EtOAc).

\section{Reduction of Alkylidenemalononitriles with $\mathrm{NaBH}_{4}$; General Pro-} cedure B

The corresponding alkylidenemalononitrile adduct ( 1 equiv.) was dissolved in $\mathrm{MeOH}(0.3 \mathrm{M})$ at $0{ }^{\circ} \mathrm{C}$ in a flame-dried Schlenk flask under $\mathrm{N}_{2}$. $\mathrm{NaBH}_{4}$ (1.5 equiv.) was then added and the reaction progress was monitored by TLC. Once completion was achieved, the excess $\mathrm{NaBH}_{4}$ was slowly quenched with $\mathrm{H}_{2} \mathrm{O}(1 \mathrm{~mL})$ and the reaction mixture was diluted with $\mathrm{H}_{2} \mathrm{O}$ ( 5 times the reaction volume). The resulting aqueous mixture was extracted with EtOAc three times ( 2 times the aqueous phase volume). The combined organic layers were washed with brine (equal volume as the organic phase) and dried over $\mathrm{Na}_{2} \mathrm{SO}_{4}$. The solvents were removed under reduced pressure and the crude was purified via silica gel column chromatography (Hex/EtOAc) or used as crude when sufficiently pure.

Oxidation of Primary or Secondary Alcohols to Aldehydes or Ketones; General Procedure C

The corresponding alcohol (1 equiv.) was dissolved in DCM ( $0.2 \mathrm{M})$ at $\mathrm{rt}$ and pyridinium chlorochromate (1.5 equiv.) was added. The reaction progress was monitored by TLC. After completion, the reaction mixture was filtered through a short pad of silica gel with elution with the corresponding Hex/EtOAc mixture. The solvents were removed under reduced pressure and the crude was purified via silica gel column chromatography (Hex/EtOAc) or used as crude when sufficiently pure.

TiCl $_{4}$-Catalyzed Allylboration of Aldehydes; General Procedure D-1 The corresponding aldehyde ( 1 equiv.) was dissolved in toluene $(0.2$ $\mathrm{M})$ at $\mathrm{rt}$ in a flame-dried Schlenk flask under $\mathrm{N}_{2}$. Allylboronic acid pinacol ester ( 1.5 equiv.) was then added dropwise at rt, followed by $\mathrm{TiCl}_{4}$ (1 mol\%). After reaction completion, the mixture was diluted with $\mathrm{H}_{2} \mathrm{O}$ (same volume as the reaction mixture) and extracted with EtOAc three times ( $1 / 3$ of the total volume each time). The combined organic layers were washed with brine (equal volume as the organic phase) and dried over $\mathrm{Na}_{2} \mathrm{SO}_{4}$. The solvents were removed under reduced pressure and the crude was purified via silica gel column chromatography (Hex/EtOAc) or used as crude when sufficiently pure.

\section{Allylboration of Aldehydes; General Procedure D-2}

The corresponding aldehyde ( 1 equiv.) was dissolved in toluene $(0.2$ $\mathrm{M})$ at $\mathrm{rt}$ in a flame-dried Schlenk flask under $\mathrm{N}_{2}$. Allylboronic acid pinacol ester (1.5 equiv.) was then added dropwise at $\mathrm{rt}$ and the reaction 
mixture was heated at $80^{\circ} \mathrm{C}$. After reaction completion, the mixture was diluted with $\mathrm{H}_{2} \mathrm{O}$ (same volume as the reaction mixture) and extracted with EtOAc three times (1/3 of the total volume each time). The combined organic layers were washed with brine (equal volume as the organic phase) and dried over $\mathrm{Na}_{2} \mathrm{SO}_{4}$. The solvents were removed under reduced pressure and the crude was purified via silica gel column chromatography (Hex/EtOAc) or used as crude when sufficiently pure.

\section{Grignard Addition to Aldehydes; General Procedure E}

The corresponding aldehyde ( 1 equiv.) was dissolved in $\operatorname{THF}(0.2 \mathrm{M})$ in a flame-dried Schlenk flask under $\mathrm{N}_{2}$. The reaction mixture was then cooled to $-78{ }^{\circ} \mathrm{C}$. Vinylmagnesium bromide solution (1.3 equiv., $1 \mathrm{M}$ in THF) was added and the reaction mixture was stirred at $-78^{\circ} \mathrm{C}$. After reaction completion, the mixture was allowed to reach $\mathrm{rt}$ and was quenched with $2 \mathrm{M} \mathrm{HCl}_{(\mathrm{aq})}$ (3 times the reaction volume). The resulting mixture was extracted 3 times with EtOAc (1/3 of the total volume, 3 times). The combined organic layers were washed with brine (equal volume as the organic phase) and dried over $\mathrm{Na}_{2} \mathrm{SO}_{4}$. The solvents were removed under reduced pressure and the crude was purified via silica gel column chromatography (Hex/EtOAc) or used as crude when sufficiently pure.

\section{Ring-Closing Metathesis; General Procedure F}

The corresponding diene (1 equiv.) was dissolved in toluene (0.05 M) in a flame-dried Schlenk flask under $\mathrm{N}_{2}$. Grubbs $2^{\text {nd }}$ generation catalyst ( $1 \mathrm{~mol} \%$ ) was then added and the reaction mixture was stirred at $80{ }^{\circ} \mathrm{C}$ overnight. After reaction completion, the toluene was removed under reduced pressure and the residue was purified via silica gel column chromatography (Hex/EtOAc).

\section{Wittig Reaction on Aldehydes; General Procedure G}

Methyltriphenylphosphonium bromide (2 equiv.) was dissolved in THF $(1 \mathrm{~mL})$ and cooled to $0{ }^{\circ} \mathrm{C}$. Potassium tert-butoxide (2.5 equiv.) was then added at $0{ }^{\circ} \mathrm{C}$ and the reaction mixture was stirred at this temperature for $15 \mathrm{~min}$. After $15 \mathrm{~min}$, the desired aldehyde (1 equiv.) in THF ( $0.4 \mathrm{~mL}, 0.1 \mathrm{M}$ final concentration) was added dropwise at $0{ }^{\circ} \mathrm{C}$. The reaction mixture was slowly allowed to reach rt overnight. After $14 \mathrm{~h}$, the reaction mixture was quenched with a saturated $\mathrm{NH}_{4} \mathrm{Cl}_{(\mathrm{aq})}$ solution $(10 \mathrm{~mL})$ and was extracted with EtOAc $(3 \times 5 \mathrm{~mL})$. The combined organic layers were washed with brine $(15 \mathrm{~mL})$ and dried over $\mathrm{Na}_{2} \mathrm{SO}_{4}$. The solvents were removed under reduced pressure and the residue was purified via silica gel column chromatography (Hex/EtOAc).

\section{2-Benzyl-3-phenylpropan-1-ol (3a)}

Prepared from general procedure A-1 (colorless oil, $54.7 \mathrm{mg}, 62 \%$ ). Solvent system for chromatography: Hex/EtOAc $15 \% .{ }^{1} \mathrm{H}$ and ${ }^{13} \mathrm{C}$ NMR data matched reported values. ${ }^{58}$

${ }^{1} \mathrm{H}$ NMR $\left(600 \mathrm{MHz}, \mathrm{CDCl}_{3}\right): \delta=7.29(\mathrm{~m}, 4 \mathrm{H}), 7.23-7.16(\mathrm{~m}, 6 \mathrm{H}), 3.50$ $(\mathrm{d}, J=4.9 \mathrm{~Hz}, 2 \mathrm{H}), 2.70(\mathrm{dd}, J=13.7,8.0 \mathrm{~Hz}, 2 \mathrm{H}), 2.66$ (dd, $J=13.7,6.5$ $\mathrm{Hz}, 2 \mathrm{H}), 2.19-2.10$ (m, $1 \mathrm{H})$.

$\left.{ }^{13} \mathrm{C} \mathrm{NMR} \mathrm{(151} \mathrm{MHz,} \mathrm{CDCl}_{3}\right): \delta=140.6,129.3,128.5,126.1,64.2,44.7$, 37.6.

\section{(1,2,3,4-Tetrahydronaphthalen-2-yl)methanol (3b)}

Prepared from general procedure A-2 (pale yellow oil, $31 \mathrm{mg}, 75 \%$ ). Solvent system for chromatography: Hex/EtOAc $20 \% .{ }^{1} \mathrm{H}$ and ${ }^{13} \mathrm{C}$ NMR data matched reported values. ${ }^{59}$
${ }^{1} \mathrm{H}$ NMR $\left(400 \mathrm{MHz}, \mathrm{CDCl}_{3}\right): \delta=7.15-7.07(\mathrm{~m}, 4 \mathrm{H}), 3.69-3.60(\mathrm{~m}, 2 \mathrm{H})$, $2.91(\mathrm{dd}, J=11.7,1.9 \mathrm{~Hz}, 1 \mathrm{H}), 2.87(\mathrm{~m}, 2 \mathrm{H}), 2.60-2.46(\mathrm{~m}, 1 \mathrm{H}), 2.06-$ 1.93 (m, 2 H), 1.90-1.78 (m, 1 H), 1.46 (tddd, $J=11.6,9.9,5.8,1.9 \mathrm{~Hz}, 1$ $\mathrm{H})$.

${ }^{13} \mathrm{C}$ NMR $\left(101 \mathrm{MHz}, \mathrm{CDCl}_{3}\right)$ : $\delta=136.9,136.0,129.3,129.0,125.7$, $125.7,67.8,37.2,32.5,28.8,26.0$.

\section{(1,2,3,4-Tetrahydronaphthalen-1-yl)methanol (3c)}

Prepared from general procedure A-1 (pale yellow oil, $44 \mathrm{mg}, 53 \%$ ). Solvent system for chromatography: Hex/EtOAc $10 \% .{ }^{1} \mathrm{H}$ and ${ }^{13} \mathrm{C}$ NMR data matched reported values. ${ }^{60}$

${ }^{1} \mathrm{H}$ NMR $\left(400 \mathrm{MHz}, \mathrm{CDCl}_{3}\right): \delta=7.25-7.08(\mathrm{~m}, 4 \mathrm{H}), 3.81(\mathrm{~d}, J=6.4 \mathrm{~Hz}, 2$ $\mathrm{H}), 3.04-2.94(\mathrm{~m}, 1 \mathrm{H}), 2.77(\mathrm{td}, J=5.8,3.5 \mathrm{~Hz}, 2 \mathrm{H}), 1.96-1.81(\mathrm{~m}, 3$ $\mathrm{H}), 1.81-1.70(\mathrm{~m}, 1 \mathrm{H}), 1.49(\mathrm{bs}, J=6.5 \mathrm{~Hz}, 1 \mathrm{H})$.

${ }^{13} \mathrm{C}$ NMR $\left(101 \mathrm{MHz}, \mathrm{CDCl}_{3}\right): \delta=138.3,136.8,129.5,128.9,126.3$, $125.8,67.3,40.4,29.9,25.3,19.9$.

\section{(4-Phenylcyclohexyl)methanol (3d)}

Prepared from general procedure A-1 (colorless oil, $56 \mathrm{mg}, 65 \%, 2.5: 1$ dr). Solvent system for chromatography: Hex/EtOAc $20 \% .{ }^{1} \mathrm{H}$ and ${ }^{13} \mathrm{C}$ NMR data matched reported values. ${ }^{61}$ See ${ }^{1} \mathrm{H}$ and ${ }^{13} \mathrm{C}$ NMR spectra of 3d in the supporting information for more details.

\section{Ethyl 4-(Hydroxymethyl)cyclohexane-1-carboxylate (3e)}

Prepared from general procedure A-1 (colorless oil, $25 \mathrm{mg}, 56 \%, 2: 1$ dr). Solvent system for chromatography: Hex/EtOAc $20 \% .{ }^{1} \mathrm{H}$ and ${ }^{13} \mathrm{C}$ NMR data matched reported values. ${ }^{62}$ See ${ }^{1} \mathrm{H}$ and ${ }^{13} \mathrm{C}$ NMR spectra of $\mathbf{3 e}$ in the supporting information for more details.

\section{((5R)-2-Methyl-5-(prop-1-en-2-yl)cyclohexyl)methanol (3f)}

Prepared from general procedure A-1 (colorless oil, $56 \mathrm{mg}, 90 \%$, 14:2:1:1 mixture of isomers, major reported). Solvent system for chromatography: Hex/EtOAc $10 \%$.

${ }^{1} \mathrm{H}$ NMR $\left(400 \mathrm{MHz}, \mathrm{CDCl}_{3}\right): \delta=4.68(\mathrm{dd}, J=1.8,1.1 \mathrm{~Hz}, 2 \mathrm{H}), 3.52(\mathrm{dd}$, $J=10.6,7.6 \mathrm{~Hz}, 1 \mathrm{H}), 3.45(\mathrm{dd}, J=10.5,7.0 \mathrm{~Hz}, 1 \mathrm{H}), 2.03(\mathrm{dq}, J=7.1$, $3.9 \mathrm{~Hz}, 1 \mathrm{H}), 1.91(\mathrm{tt}, J=12.1,3.4 \mathrm{~Hz}, 1 \mathrm{H}), 1.78$ (ddd, $J=12.6,7.9,3.7$ $\mathrm{Hz}, 1 \mathrm{H}), 1.72(\mathrm{~s}, 3 \mathrm{H}), 1.65-1.59(\mathrm{~m}, 1 \mathrm{H}), 1.57$ (q, J = 3.6 Hz, $1 \mathrm{H})$, $1.55-1.49(\mathrm{~m}, 2 \mathrm{H}), 1.40(\mathrm{dd}, J=13.2,4.4 \mathrm{~Hz}, 1 \mathrm{H}), 1.36-1.31(\mathrm{~m}, 1 \mathrm{H})$, 1.09 (q, J = 12.6 Hz, $1 \mathrm{H}), 0.84$ (d, J = 7.2 Hz, $3 \mathrm{H}$ ).

${ }^{13} \mathrm{C}$ NMR $\left(101 \mathrm{MHz}, \mathrm{CDCl}_{3}\right): \delta=150.8,108.3,66.5,45.4,43.3,33.4$, 28.5, 28.3, 25.9, 21.1, 12.1 .

HRMS (ESI-TOF): $m / z\left[\mathrm{M}+\mathrm{NH}_{4}\right]^{+}$calcd for $\mathrm{C}_{11} \mathrm{H}_{24} \mathrm{NO}$ : 186.1552; found: 186.1556 .

\section{tert-Butyl (1R,3S,5S)-3-(Hydroxymethyl)-8-azabicyclo[3.2.1]oc-} tane-8-carboxylate (3g)

Prepared from general procedure A-1 (pale yellow oil, $20 \mathrm{mg}, 45 \%$, $11: 1 \mathrm{dr}$ ). Solvent system for chromatography: Hex/EtOAc 30\% to $40 \%$. ${ }^{1} \mathrm{H}$ and ${ }^{13} \mathrm{C}$ NMR data matched reported values. ${ }^{47}$

${ }^{1} \mathrm{H} \mathrm{NMR}\left(400 \mathrm{MHz}\right.$, toluene- $\left.d_{8}, 80^{\circ} \mathrm{C}\right): \delta=4.21(\mathrm{bs}, 2 \mathrm{H}), 3.10(\mathrm{~d}, J=6.0$ $\mathrm{Hz}, 2 \mathrm{H}), 1.79-1.58(\mathrm{~m}, 4 \mathrm{H}), 1.46(\mathrm{~s}, 9 \mathrm{H}), 1.43-1.37$ (m, $2 \mathrm{H}), 1.37-$ $1.32(\mathrm{~m}, 2 \mathrm{H}), 1.32-1.27(\mathrm{~m}, 2 \mathrm{H})$.

${ }^{13} \mathrm{C}$ NMR (101 MHz, toluene- $\left.d_{8}, 80{ }^{\circ} \mathrm{C}\right): \delta=153.0,78.0,67.5,53.3,33.8$, 31.3, 28.2, 28.0.

HRMS (ESI-TOF): $m / z$ [M $+\mathrm{H}]^{+}$calcd for $\mathrm{C}_{13} \mathrm{H}_{24} \mathrm{NO}_{3}: 242.1761$; found: 242.1750. 


\section{3-(3,4-Dimethoxyphenyl)-2-methylpropan-1-ol (3h)}

Prepared from general procedure A-1 (colorless oil, $52 \mathrm{mg}, 60 \%$ ). Solvent system for chromatography: Hex/EtOAc 30\%. ${ }^{1} \mathrm{H}$ and ${ }^{13} \mathrm{C}$ NMR data matched reported values. ${ }^{63}$

${ }^{1} \mathrm{H}$ NMR $\left(400 \mathrm{MHz}, \mathrm{CDCl}_{3}\right): \delta=6.82-6.76(\mathrm{~m}, 1 \mathrm{H}), 6.73-6.68(\mathrm{~m}, 2 \mathrm{H})$, $3.86(\mathrm{dd}, J=3.8,0.9 \mathrm{~Hz}, 6 \mathrm{H}), 3.53(\mathrm{ddt}, J=10.5,5.9,0.9 \mathrm{~Hz}, 1 \mathrm{H}), 3.48$ (ddt, $J=10.4,6.0,0.9 \mathrm{~Hz}, 1 \mathrm{H}$ ), 2.70 (dd, $J=13.5,6.4 \mathrm{~Hz}, 1 \mathrm{H}), 2.38$ (dd, $J=13.6,8.0 \mathrm{~Hz}, 1 \mathrm{H}), 2.00-1.85(\mathrm{~m}, 1 \mathrm{H}), 1.42(\mathrm{bs}, 1 \mathrm{H}), 0.92(\mathrm{dd}, J=$ $6.8,1.0 \mathrm{~Hz}, 3 \mathrm{H})$

${ }^{13} \mathrm{C}$ NMR $\left(101 \mathrm{MHz}, \mathrm{CDCl}_{3}\right): \delta=148.9,147.4,133.3,121.1,112.4$, $111.19,67.9,56.0,39.5,38.0,16.7$.

\section{2-(Pyridin-3-yl)ethan-1-ol (3i)}

Prepared from general procedure A-1 (yellow oil, $36 \mathrm{mg}, 20 \%$, impure). Solvent system for chromatography: Hex/EtOAc $40 \% .{ }^{1} \mathrm{H}$ and ${ }^{13} \mathrm{C}$ NMR data matched reported values. ${ }^{64}$

${ }^{1} \mathrm{H} \mathrm{NMR}\left(600 \mathrm{MHz}, \mathrm{CDCl}_{3}\right): \delta=8.47(\mathrm{~s}, 1 \mathrm{H}), 8.42(\mathrm{~d}, J=5.8 \mathrm{~Hz}, 1 \mathrm{H})$, $7.82(\mathrm{dt}, J=7.8,1.6 \mathrm{~Hz}, 1 \mathrm{H}), 7.43(\mathrm{dd}, J=7.9,5.7 \mathrm{~Hz}, 1 \mathrm{H}), 3.87(\mathrm{t}, J=$ $6.2 \mathrm{~Hz}, 2 \mathrm{H}), 2.89(\mathrm{t}, J=6.2 \mathrm{~Hz}, 2 \mathrm{H})$.

${ }^{13} \mathrm{C}$ NMR $\left(151 \mathrm{MHz}, \mathrm{CDCl}_{3}\right): \delta=147.8,145.4,140.0,137.6,125.1,62.1$, 35.8.

\section{2-Cyclopropylhexa-4,5-dien-1-ol (3j)}

Prepared from general procedure A-2 (colorless oil, $2 \mathrm{mg}, 11 \%$ ). Solvent system for chromatography: Hex/EtOAc $5 \%$ to $20 \%$.

${ }^{1} \mathrm{H}$ NMR $\left(600 \mathrm{MHz}, \mathrm{CDCl}_{3}\right): \delta=5.15(\mathrm{dq}, J=7.9,6.8 \mathrm{~Hz}, 1 \mathrm{H}), 4.66(\mathrm{dt}$, $J=6.8,2.8 \mathrm{~Hz}, 2 \mathrm{H}), 3.68(\mathrm{~d}, J=5.7 \mathrm{~Hz}, 2 \mathrm{H}), 2.25-2.13(\mathrm{~m}, 2 \mathrm{H}), 1.50$ (bs, $1 \mathrm{H}), 0.87$ (ddq, $J=9.5,7.3,5.8 \mathrm{~Hz}, 1 \mathrm{H}), 0.65-0.56(\mathrm{~m}, 1 \mathrm{H}), 0.52-$ $0.47(\mathrm{~m}, 2 \mathrm{H}), 0.23-0.17(\mathrm{~m}, 1 \mathrm{H}), 0.16-0.12(\mathrm{~m}, 1 \mathrm{H})$.

${ }^{13} \mathrm{C}$ NMR $\left(151 \mathrm{MHz}, \mathrm{CDCl}_{3}\right): \delta=209.1,88.0,74.5,66.4,46.5,31.1,13.1$, 3.8, 3.4 .

\section{5-(2-Hydroxyethyl)-2-methoxyphenol (3k)}

Prepared from general procedure A-1 (pale yellow oil, $21 \mathrm{mg}, 22 \%$ ). Solvent system for chromatography: Hex/EtOAc 30\%. ${ }^{1} \mathrm{H}$ and ${ }^{13} \mathrm{C}$ NMR data matched reported values. ${ }^{65}$

${ }^{1} \mathrm{H} \mathrm{NMR}\left(400 \mathrm{MHz}, \mathrm{CDCl}_{3}\right): \delta=6.81(\mathrm{~s}, 1 \mathrm{H}), 6.79(\mathrm{~d}, J=6.0 \mathrm{~Hz}, 1 \mathrm{H})$, $6.70(\mathrm{dd}, J=8.2,2.1 \mathrm{~Hz}, 1 \mathrm{H}), 3.87(\mathrm{~s}, 3 \mathrm{H}), 3.82(\mathrm{t}, J=6.5 \mathrm{~Hz}, 2 \mathrm{H}), 2.77$ $(\mathrm{t}, J=6.5 \mathrm{~Hz}, 2 \mathrm{H})$.

${ }^{13} \mathrm{C}$ NMR $\left(101 \mathrm{MHz}, \mathrm{CDCl}_{3}\right): \delta=145.8,145.4,131.7,120.6,115.2$, $110.9,63.8,56.1,38.6$.

\section{(1-Benzylpiperidin-4-yl)methanol (31)}

Prepared from general procedure A-1 (colorless oil, $72 \mathrm{mg}, 17 \%$ ). Solvent system for chromatography: Hex/EtOAc $20 \% .{ }^{1} \mathrm{H}$ and ${ }^{13} \mathrm{C}$ NMR data matched reported values. ${ }^{66}$

${ }^{1} \mathrm{H}$ NMR (400 MHz, methanol- $\left.d_{4}\right): \delta=7.44(\mathrm{dd}, J=6.7,3.0 \mathrm{~Hz}, 2 \mathrm{H})$, 7.39-7.35 (m, $3 \mathrm{H}), 4.01(\mathrm{~s}, 2 \mathrm{H}), 3.40(\mathrm{~d}, J=6.5 \mathrm{~Hz}, 2 \mathrm{H}), 2.93$ (dt, $J=$ 11.9, $2.3 \mathrm{~Hz}, 2 \mathrm{H}$ ), 2.61 (ddd, $J=13.4,11.9,3.2 \mathrm{~Hz}, 2 \mathrm{H}$ ), 1.99 (qd, $J=$ 13.0, $3.8 \mathrm{~Hz}, 2 \mathrm{H}$ ), 1.56 (ddt, $J=13.9,3.5,1.7 \mathrm{~Hz}, 2 \mathrm{H}$ ), 1.36 (dddd, $J=$ 16.6, 12.3, 6.6, $2.4 \mathrm{~Hz}, 1 \mathrm{H})$.

${ }^{13} \mathrm{C}$ NMR $\left(101 \mathrm{MHz}\right.$, methanol- $\left.d_{4}\right): \delta=134.4,132.3,129.8,129.0,71.2$, $67.5,57.6,38.7,25.4$.

\section{3,3-Diallyl-4-methyldihydrofuran-2(3H)-one (3m)}

Prepared from general procedure A-1 (colorless oil, $90 \mathrm{mg}, 65 \%$ ). Solvent system for chromatography: Hex/EtOAc 5\%.
${ }^{1} \mathrm{H} \mathrm{NMR}\left(400 \mathrm{MHz}, \mathrm{CDCl}_{3}\right): \delta=5.88-5.77(\mathrm{~m}, 1 \mathrm{H}), 5.77-5.66(\mathrm{~m}, 1 \mathrm{H})$, 5.18-5.14 (m, $2 \mathrm{H}), 5.14-5.07(\mathrm{~m}, 2 \mathrm{H}), 4.29(\mathrm{dd}, J=8.9,8.0 \mathrm{~Hz}, 1 \mathrm{H})$, 3.80 (dd, $J=10.0,8.9 \mathrm{~Hz}, 1 \mathrm{H}$ ), 2.59 (ddq, $J=10.0,8.1,7.1 \mathrm{~Hz}, 1 \mathrm{H}$ ), 2.45 (ddt, $J=14.1,6.3,1.3 \mathrm{~Hz}, 1 \mathrm{H}), 2.34(\mathrm{ddt}, J=14.2,7.2,1.3 \mathrm{~Hz}, 1 \mathrm{H}), 2.24$ (ddt, $J=12.9,8.5,0.8 \mathrm{~Hz}, 1 \mathrm{H}$ ), 2.19 (ddt $J=13.1,7.6,1.1 \mathrm{~Hz}, 1 \mathrm{H}$ ), 1.05 $(\mathrm{d}, J=7.0 \mathrm{~Hz}, 3 \mathrm{H})$.

${ }^{13} \mathrm{C}$ NMR $\left(101 \mathrm{MHz}, \mathrm{CDCl}_{3}\right): \delta=180.0,133.1,132.8,119.5,119.4,71.4$, 48.3, 39.3, 37.0, 35.9, 11.2.

HRMS (ESI-TOF): $m / z$ [M+ H] $]^{+}$calcd for $\mathrm{C}_{11} \mathrm{H}_{17} \mathrm{O}_{2}: 181.1223$; found: 181.1218

(1S,2S,3R)-3-(Hydroxymethyl)-2-methyl-2-(prop-2-yn-1-yl)cyclopentan-1-ol (3n)

Prepared from general procedure A-2 (pale yellow oil, $18 \mathrm{mg}, 22 \%$, $20: 1 \mathrm{dr})$. Solvent system for chromatography: Hex/EtOAc $20 \%$ to $30 \%$.

${ }^{1} \mathrm{H}$ NMR $\left(400 \mathrm{MHz}, \mathrm{CDCl}_{3}\right): \delta=3.79(\mathrm{dd}, J=11.1,2.6 \mathrm{~Hz}, 1 \mathrm{H}), 3.75(\mathrm{dd}$, $J=5.8,2.1 \mathrm{~Hz}, 1 \mathrm{H}), 3.55(\mathrm{dd}, J=11.1,3.7 \mathrm{~Hz}, 1 \mathrm{H}), 2.37(\mathrm{t}, J=2.9 \mathrm{~Hz}, 2$ H), 2.12-2.03 (m, $1 \mathrm{H}), 1.99(\mathrm{t}, J=2.6 \mathrm{~Hz}, 1 \mathrm{H}), 1.94-1.86(\mathrm{~m}, 1 \mathrm{H}), 1.81$ (dddd, $J=11.3,9.6,6.5,3.1 \mathrm{~Hz}, 1 \mathrm{H}$ ), 1.65 (dddd, $J=13.4,9.7,3.4,1.7$ $\mathrm{Hz}, 1 \mathrm{H}), 1.11(\mathrm{~s}, 3 \mathrm{H})$.

${ }^{13} \mathrm{C}$ NMR $\left(101 \mathrm{MHz}, \mathrm{CDCl}_{3}\right): \delta=83.1,80.0,70.1,63.1,48.2,48.0,32.4$, $26.1,24.3,22.1$.

((7S,8S)-7-((R,E)-1,3-Diphenylallyl)-1,4-dioxaspiro[4.5]decan-8yl)methanol (4a)

Prepared from general procedure A-1 (white foam, $265 \mathrm{mg}$ and $72 \mathrm{mg}$ for $\mathbf{4 a}$ and iso-4a respectively, 53\%, 3.5:1 dr, separable diastereomers, major reported). Solvent system for chromatography: Hex/EtOAc 25\% to $30 \%$. Characterization of iso-4a is available in the Supporting Information.

${ }^{1} \mathrm{H}$ NMR (400 MHz, $\mathrm{CDCl}_{3}$ ): $\delta=7.34-7.24(\mathrm{~m}, 6 \mathrm{H}), 7.23-7.15(\mathrm{~m}, 4 \mathrm{H})$, $6.45(\mathrm{~d}, J=15.8 \mathrm{~Hz}, 1 \mathrm{H}), 6.34(\mathrm{dd}, J=15.8,9.2 \mathrm{~Hz}, 1 \mathrm{H}), 3.95(\mathrm{dd}, J=$ 10.7, $4.7 \mathrm{~Hz}, 1 \mathrm{H}), 3.90-3.80(\mathrm{~m}, 2 \mathrm{H}), 3.79-3.64(\mathrm{~m}, 3 \mathrm{H}), 3.27$ (dd, $J=$ 11.7, $9.2 \mathrm{~Hz}, 1 \mathrm{H}), 2.36$ (tt, $J=12.0,4.3 \mathrm{~Hz}, 1 \mathrm{H}), 2.14(\mathrm{dt}, J=9.4,4.2 \mathrm{~Hz}$, $1 \mathrm{H}), 2.07(\mathrm{dt}, J=9.9,2.6 \mathrm{~Hz}, 1 \mathrm{H}), 1.71-1.63(\mathrm{~m}, 2 \mathrm{H}), 1.64-1.57$ (m, 1 H), $1.35-1.17$ (m, $3 \mathrm{H})$.

${ }^{13} \mathrm{C}$ NMR $\left(101 \mathrm{MHz}, \mathrm{CDCl}_{3}\right): \delta=143.8,137.6,133.2,130.6,129.2$, 128.9, 128.1, 127.7, 126.8, 126.6, 109.5, 64.5, 64.5, 59.1, 53.0, 42.5, $37.1,35.2,30.4,24.8$.

(S)-3-((1R,3R,5S)-6,6-Dimethyl-2-methylenebicyclo[3.1.1]heptan3-yl)-4-phenylbutan-1-ol (4b)

Prepared from general procedure A-2 (colorless oil, $60 \mathrm{mg}, 67 \%,>20: 1$ dr). Solvent system for chromatography: Hex/EtOAc $10 \%$.

${ }^{1} \mathrm{H}$ NMR $\left(400 \mathrm{MHz}, \mathrm{CDCl}_{3}\right)$ : $\delta=7.30-7.26(\mathrm{~m}, 2 \mathrm{H}), 7.20-7.14(\mathrm{~m}, 3 \mathrm{H})$, $4.93(\mathrm{t}, J=1.8 \mathrm{~Hz}, 1 \mathrm{H}), 4.87$ (dd, $J=2.5,1.4 \mathrm{~Hz}, 1 \mathrm{H}$ ), 3.58 (ddd, $J$ = 10.5, 7.4, $5.8 \mathrm{~Hz}, 1 \mathrm{H}$ ), 3.47 (dt, $J=10.5,7.3 \mathrm{~Hz}, 1 \mathrm{H}$ ), 3.00-2.87 (m, 2 $\mathrm{H}), 2.54(\mathrm{t}, J=5.6 \mathrm{~Hz}, 1 \mathrm{H}), 2.36(\mathrm{dtd}, J=9.9,6.0,2.0 \mathrm{~Hz}, 1 \mathrm{H}), 2.26$ (ddd, $J=8.3,4.6,2.1 \mathrm{~Hz}, 1 \mathrm{H}), 2.19(\mathrm{t}, J=11.8 \mathrm{~Hz}, 1 \mathrm{H}$ ), $2.05(\mathrm{tt}, J=5.9$, $2.9 \mathrm{~Hz}, 1 \mathrm{H}$ ), 1.96 (dddd, $J=13.9,10.6,3.3,1.9 \mathrm{~Hz}, 1 \mathrm{H}$ ), 1.74 (ddd, $J=$ 13.7, 5.8, $2.9 \mathrm{~Hz}, 1 \mathrm{H}$ ), 1.60-1.48 (m, $2 \mathrm{H}), 1.29$ (s, $3 \mathrm{H}$ ), 1.21 (d, J = 9.9 $\mathrm{Hz}, 1 \mathrm{H}), 0.88(\mathrm{~s}, 3 \mathrm{H})$.

${ }^{13} \mathrm{C}$ NMR $\left(101 \mathrm{MHz}, \mathrm{CDCl}_{3}\right): \delta=154.8,141.9,129.4,128.4,125.9$, 107.4, 61.7, 53.4, 41.6, 41.1, 40.4, 36.9, 36.1, 33.6, 29.2, 26.4, 22.1.

HRMS (ESI-TOF): $m / z$ [M $+\mathrm{H}]^{+}$calcd for $\mathrm{C}_{20} \mathrm{H}_{29} \mathrm{O}: 285.2213$; found: 285.2220 . 


\section{(3S,4S)-3-Benzyl-4-phenylhex-5-en-1-ol (4c)}

Prepared from general procedure A-2 (pale yellow oil, $44 \mathrm{mg}, 59 \%$, 10:1 dr, major reported). Solvent system for chromatography: Hex/EtOAc $10 \%$.

${ }^{1} \mathrm{H} \mathrm{NMR}\left(400 \mathrm{MHz}, \mathrm{CDCl}_{3}\right): \delta=7.26(\mathrm{~m}, 5 \mathrm{H}), 7.21-7.12(\mathrm{~m}, 5 \mathrm{H}), 6.08$ (ddd, $J=17.0,10.2,9.3 \mathrm{~Hz}, 1 \mathrm{H}), 5.19(\mathrm{dd}, J=10.2,1.8 \mathrm{~Hz}, 1 \mathrm{H}), 5.12$ (ddd, $J=17.0,1.8,0.9 \mathrm{~Hz}, 1 \mathrm{H}$ ), 3.60-3.48 (m, $2 \mathrm{H}$ ), 3.30 (dd, $J=9.4,6.7$ $\mathrm{Hz}, 1 \mathrm{H}$ ), 2.65 (dd, $J=13.8,6.2 \mathrm{~Hz}, 1 \mathrm{H}), 2.46$ (dd, $J=13.8,8.0 \mathrm{~Hz}, 1 \mathrm{H}$ ), 2.30-2.19 (m, $1 \mathrm{H}$ ), 1.73 (dddd, $J=13.9,7.8,7.0,4.3 \mathrm{~Hz}, 1 \mathrm{H}$ ), 1.59$1.49(\mathrm{~m}, 1 \mathrm{H}), 0.95$ (bs, $1 \mathrm{H})$.

${ }^{13} \mathrm{C}$ NMR $\left(101 \mathrm{MHz}, \mathrm{CDCl}_{3}\right): \delta=143.8,141.1,139.2,129.2,128.6$, $128.5,128.1,126.4,126.1,117.0,61.3,53.3,42.0,38.7,33.6$.

\section{(2S,3S)-3-Benzyl-5-hydroxy-2-vinylpentyl Acetate (4d)}

Prepared from general procedure A-2 (colorless oil, $17.5 \mathrm{mg}, 39 \%, 2: 1$ $\mathrm{dr}$, major reported). Solvent system for chromatography: Hex/EtOAc $20 \%$ to $30 \%$.

$\left.{ }^{1} \mathrm{H} \mathrm{NMR} \mathrm{(400} \mathrm{MHz,} \mathrm{CDCl}_{3}\right): \delta=7.31-7.12(\mathrm{~m}, 5 \mathrm{H}), 5.74$ (ddd, $J=17.2$, $10.4,8.6 \mathrm{~Hz}, 1 \mathrm{H}), 5.23-5.17(\mathrm{~m}, 1 \mathrm{H}), 5.15-5.09(\mathrm{~m}, 1 \mathrm{H}), 4.16(\mathrm{dd}, J=$ 7.1, 3.7 Hz, $1 \mathrm{H}$ ), 4.09 (d, J = 7.2 Hz, $1 \mathrm{H}), 3.61-3.52(\mathrm{~m}, 2 \mathrm{H}), 2.80$ (dd, $J=13.7,5.1 \mathrm{~Hz}, 1 \mathrm{H}), 2.63-2.56(\mathrm{~m}, 1 \mathrm{H}), 2.38(\mathrm{dd}, J=13.7,9.5 \mathrm{~Hz}, 1 \mathrm{H})$, 2.06 (s, $3 \mathrm{H}), 1.64-1.46(\mathrm{~m}, 2 \mathrm{H})$.

${ }^{13} \mathrm{C}$ NMR (101 MHz, $\left.\mathrm{CDCl}_{3}\right): \delta=171.2,140.9,136.2,129.2,128.5$, $126.2,118.3,65.4,60.9,45.1,38.0,37.1,34.0,21.1$.

1-((7S,8S)-7-((R,E)-1,3-Diphenylallyl)-1,4-dioxaspiro[4.5]decan-8yl)but-3-en-1-ol (5a-SI-2 and iso-5a-SI-2)

Prepared from $4 \mathrm{a}$ via general procedure $\mathrm{C}$ followed by general procedure D-2 (white foam, $15 \mathrm{mg}$ and $32 \mathrm{mg}$ respectively, $80 \%$ over 2 steps, 2:1 dr). The intermediate after general procedure $C$ was used crude without further purification. As the newly formed stereocenter will be obliterated later in the sequence, the stereochemical assignment was not determined. Solvent system for chromatography: Hex/EtOAc 15\%.

\section{Diastereomer 1}

${ }^{1} \mathrm{H} \mathrm{NMR}\left(400 \mathrm{MHz}, \mathrm{CDCl}_{3}\right): \delta=7.39-7.27(\mathrm{~m}, 6 \mathrm{H}), 7.25-7.13(\mathrm{~m}, 4 \mathrm{H})$, 6.51-6.37 (m, $2 \mathrm{H}$ ), 5.83 (dddd, $J=19.9,9.7,8.0,6.3 \mathrm{~Hz}, 1 \mathrm{H}), 5.16$ (d, $J=12.3 \mathrm{~Hz}, 2 \mathrm{H}), 4.11-3.99(\mathrm{~m}, 2 \mathrm{H}), 3.89-3.80(\mathrm{~m}, 2 \mathrm{H}), 3.77-3.70(\mathrm{~m}$, $1 \mathrm{H}), 3.69-3.61(\mathrm{~m}, 1 \mathrm{H}), 2.56-2.39(\mathrm{~m}, 2 \mathrm{H}), 2.12(\mathrm{dt}, J=13.9,8.2 \mathrm{~Hz}$, $1 \mathrm{H}), 1.99(\mathrm{dd}, J=9.3,4.2 \mathrm{~Hz}, 1 \mathrm{H}), 1.87-1.78(\mathrm{~m}, 1 \mathrm{H}), 1.74-1.65(\mathrm{~m}, 1$ H), 1.63-1.52 (m, $3 \mathrm{H}), 1.41-1.33(\mathrm{~m}, 1 \mathrm{H})$.

${ }^{13} \mathrm{C}$ NMR $\left(101 \mathrm{MHz}, \mathrm{CDCl}_{3}\right): \delta=144.8,137.8,135.3,134.7,128.8$, 128.6, 128.1, 127.1, 127.1, 126.2, 126.2, 119.0, 109.3, 69.3, 64.4, 64.0, 51.5, 40.9 (overlapping signals), 34.8, 32.5, 24.8 .

\section{Diastereomer 2}

${ }^{1} \mathrm{H}$ NMR $\left(400 \mathrm{MHz}, \mathrm{CDCl}_{3}\right): \delta=7.37-7.26(\mathrm{~m}, 7 \mathrm{H}), 7.25-7.15(\mathrm{~m}, 3 \mathrm{H})$, $6.43(\mathrm{~d}, J=15.7 \mathrm{~Hz}, 1 \mathrm{H}), 6.29$ (dd, $J=15.8,9.5 \mathrm{~Hz}, 1 \mathrm{H}), 5.74$ (dddd, $J=$ $16.2,10.1,8.2,6.1 \mathrm{~Hz}, 1 \mathrm{H}), 5.16-5.06(\mathrm{~m}, 2 \mathrm{H}), 4.22$ (ddd, $J=8.9,4.0$, $2.5 \mathrm{~Hz}, 1 \mathrm{H}), 3.90-3.80$ (m, $2 \mathrm{H}$ ), 3.77-3.66 (m, $2 \mathrm{H}$ ), 3.61 (dd, $J=11.6$, $9.4 \mathrm{~Hz}, 1 \mathrm{H}), 2.41(\mathrm{tt}, J=12.1,3.9 \mathrm{~Hz}, 1 \mathrm{H}), 2.34-2.20(\mathrm{~m}, 2 \mathrm{H}), 2.15(\mathrm{td}$, $J=13.2,4.6 \mathrm{~Hz}, 1 \mathrm{H}), 2.04(\mathrm{dt}, J=14.1,2.2 \mathrm{~Hz}, 1 \mathrm{H}), 1.89(\mathrm{t}, J=12.7 \mathrm{~Hz}$, $1 \mathrm{H}), 1.84(\mathrm{~d}, J=5.3 \mathrm{~Hz}, 1 \mathrm{H}), 1.70(\mathrm{tt}, J=13.7,4.9 \mathrm{~Hz}, 1 \mathrm{H}), 1.63-1.52$ (m, $2 \mathrm{H})$.

${ }^{13} \mathrm{C}$ NMR $\left(101 \mathrm{MHz}, \mathrm{CDCl}_{3}\right): \delta=144.0,137.5,135.6,133.9,130.3$, 128.8, 128.6, 128.0, 127.2, 126.3, 126.2, 118.6, 110.1, 70.1, 64.1, 64.0, $52.3,42.9,41.8,35.5,32.7,23.7$.
(4aS,9R,9aS)-9-Phenyl-3,4,4a,6,9,9a-hexahydrospiro[benzo[7]annulene-2,2'-[1,3]dioxolan]-5(1H)-one (5a)

Prepared from 5a-SI-2 and iso-5a-SI-2 mixture via general procedure F followed by general procedure C (white solid, $15 \mathrm{mg}, 41 \%$ over 2 steps, 11:1 inseparable mixture of $\alpha, \beta$ - and $\gamma, \beta$-unsaturated ketone, major reported). The intermediate after general procedure $F$ was used crude without further purification. Solvent system for chromatography: Hex/EtOAc $5 \%$.

${ }^{1} \mathrm{H}$ NMR $\left(600 \mathrm{MHz}, \mathrm{CDCl}_{3}\right): \delta=7.32(\mathrm{t}, J=7.3 \mathrm{~Hz}, 2 \mathrm{H}), 7.24(\mathrm{~m}, 3 \mathrm{H}, J=$ 5.8, 5.4, $2.8 \mathrm{~Hz}, 3 \mathrm{H}$ ), 5.91 (ddd, $J=10.3,6.0,2.3 \mathrm{~Hz}, 1 \mathrm{H}$ ), 5.85 (dddd, $J=10.5,7.2,3.7,1.8 \mathrm{~Hz}, 1 \mathrm{H}), 3.86(\mathrm{td}, J=6.6,6.2 \mathrm{~Hz}, 1 \mathrm{H}), 3.80(\mathrm{dt}, J=$ 7.0, $5.9 \mathrm{~Hz}, 1 \mathrm{H}), 3.78-3.73(\mathrm{~m}, 1 \mathrm{H}), 3.67(\mathrm{dt}, J=7.1,6.1 \mathrm{~Hz}, 1 \mathrm{H})$, 3.39-3.34 (m, $1 \mathrm{H}), 3.29$ (dd, $J=11.5,5.8 \mathrm{~Hz}, 1 \mathrm{H}), 3.18-3.12(\mathrm{~m}, 1 \mathrm{H})$, 3.06 (dd, $J=19.6,7.1 \mathrm{~Hz}, 1 \mathrm{H}), 2.62$ (tdd, $J=11.4,6.7,3.9 \mathrm{~Hz}, 1 \mathrm{H}), 2.16$ (td, $J=13.0,4.6 \mathrm{~Hz}, 1 \mathrm{H}), 2.02-1.95(\mathrm{~m}, 1 \mathrm{H}), 1.65(\mathrm{tt}, J=13.5,4.8 \mathrm{~Hz}, 1$ $\mathrm{H}), 1.58$ (ddt, $J=12.3,4.8,2.8 \mathrm{~Hz}, 1 \mathrm{H}$ ), 1.49 (ddd, $J=13.5,3.9,2.4 \mathrm{~Hz}$, $1 \mathrm{H}), 1.35$ (dd, $J=13.4,11.7 \mathrm{~Hz}, 1 \mathrm{H}$ ).

${ }^{13} \mathrm{C}$ NMR $\left(151 \mathrm{MHz}, \mathrm{CDCl}_{3}\right): \delta=212.3,143.4,136.3,128.8,128.3$, $126.8,124.2,109.0,64.3,64.2,50.3,45.9,44.5,44.1,37.3,31.6,29.9$, 23.7.

HRMS (ESI-TOF): $m / z$ [M $+\mathrm{H}]^{+}$calcd for $\mathrm{C}_{19} \mathrm{H}_{23} \mathrm{O}_{3}: 299.1642$; found: 299.1632.

(4aS,8R,8aS)-8-Phenyl-4,4a,8,8a-tetrahydro-1H-spiro[naphthalene-2,2'-[1,3]dioxolan]-5(3H)-one (6a)

Prepared from 4a via successive general procedures C, E, F followed by $C$ again. All intermediates were used crude without further purification. The intermediate after procedure $\mathrm{F}$ was purified via silica gel column chromatography (Hex/EtOAc 20\%) but not characterized (yellow oil, $18 \mathrm{mg}, 48 \%$ over 3 steps, $3: 1 \mathrm{dr}$ ). Final compound $\mathbf{6 a}$ was obtained after final treatment via general procedure C [white solid, $10 \mathrm{mg}, 80 \%$ (or 38\% over 4 steps), single diastereomer]. Solvent system for chromatography: Hex/EtOAc 5\%.

${ }^{1} \mathrm{H} \mathrm{NMR}\left(600 \mathrm{MHz}, \mathrm{CDCl}_{3}\right): \delta=7.35(\mathrm{t}, J=7.6 \mathrm{~Hz}, 2 \mathrm{H}), 7.30-7.26(\mathrm{~m}, 3$ $\mathrm{H}), 6.84(\mathrm{dd}, J=10.1,3.8 \mathrm{~Hz}, 1 \mathrm{H}), 6.15(\mathrm{dd}, J=10.0,2.0 \mathrm{~Hz}, 1 \mathrm{H}), 4.02-$ 3.96 (m, 2 H), 3.94-3.89 (m, $3 \mathrm{H}$ ), 2.60-2.51 (m, $2 \mathrm{H}), 2.22-2.10$ (m, 1 $\mathrm{H}), 1.72(\mathrm{tdd}, J=12.4,9.1,3.0 \mathrm{~Hz}, 2 \mathrm{H}), 1.66-1.54(\mathrm{~m}, 3 \mathrm{H})$.

${ }^{13} \mathrm{C}$ NMR $\left(151 \mathrm{MHz}, \mathrm{CDCl}_{3}\right): \delta=201.3,151.0,141.4,128.8,128.7$, $128.7,127.2,108.8,64.7,64.2,45.0,44.8,42.6,37.0,33.4,23.0$.

HRMS (ESI-TOF): $m / z[\mathrm{M}+\mathrm{H}]^{+}$calcd for $\mathrm{C}_{18} \mathrm{H}_{21} \mathrm{O}_{3}: 285.1485$; found: 285.1480 .

(7R,8R)-7-((R,E)-1,3-Diphenylallyl)-8-vinyl-1,4-dioxaspiro[4.5]decane (7a-SI-2)

Prepared from 4a via general procedure $\mathrm{C}$ followed by general procedure G (colorless oil, $11 \mathrm{mg}, 22 \%, 94 \%$ brsm, >20:1 dr). Solvent system for chromatography: Hex/EtOAc $5 \%$ to $10 \%$.

${ }^{1} \mathrm{H}$ NMR $\left(600 \mathrm{MHz}, \mathrm{CDCl}_{3}\right): \delta=7.32-7.26(\mathrm{~m}, 6 \mathrm{H}), 7.23-7.15(\mathrm{~m}, 4 \mathrm{H})$, $6.35(\mathrm{~d}, J=15.7 \mathrm{~Hz}, 1 \mathrm{H}), 6.25(\mathrm{dd}, J=15.7,9.2 \mathrm{~Hz}, 1 \mathrm{H}), 6.13(\mathrm{ddd}, J=$ 17.0, 10.3, $9.2 \mathrm{~Hz}, 1 \mathrm{H}), 5.21(\mathrm{dd}, J=10.3,2.1 \mathrm{~Hz}, 1 \mathrm{H}), 5.14(\mathrm{dd}, J=$ $17.1,1.6 \mathrm{~Hz}, 1 \mathrm{H}), 3.91-3.82(\mathrm{~m}, 2 \mathrm{H}), 3.79-3.71(\mathrm{~m}, 2 \mathrm{H}), 3.19(\mathrm{dd}, J=$ $11.3,9.2 \mathrm{~Hz}, 1 \mathrm{H}), 2.69$ (dd, $J=9.0,3.9 \mathrm{~Hz}, 1 \mathrm{H}), 2.24(\mathrm{ddt}, J=12.8,11.3$, $3.8 \mathrm{~Hz}, 1 \mathrm{H}), 1.87$ (tt, $J=14.9,4.5 \mathrm{~Hz}, 1 \mathrm{H}), 1.77-1.69(\mathrm{~m}, 2 \mathrm{H}), 1.58$ (ddt, $J=13.0,4.6,2.5 \mathrm{~Hz}, 1 \mathrm{H}), 1.33(\mathrm{t}, J=13.2 \mathrm{~Hz}, 1 \mathrm{H}), 1.30-1.26(\mathrm{~m}$, $1 \mathrm{H})$.

${ }^{13} \mathrm{C}$ NMR $\left(151 \mathrm{MHz}, \mathrm{CDCl}_{3}\right): \delta=143.4,137.7,136.3,132.8,130.5$, $128.8,128.6,128.0,127.1,126.3,126.2,117.5,109.5,64.2,64.2,53.1$, $42.4,39.6,34.7,30.5,30.2$. 
(3R,3aR,7aR)-3-Phenyl-3,3a,4,6,7,7a-hexahydrospiro[indene-5,2'[1,3]dioxolane] (7a)

Prepared from 7a-SI-2 via general procedure F (colorless oil, $5 \mathrm{mg}$, $64 \%)$. Solvent system for chromatography: Hex/EtOAc $5 \%$.

${ }^{1} \mathrm{H}$ NMR $\left(600 \mathrm{MHz}, \mathrm{CDCl}_{3}\right): \delta=7.29(\mathrm{dd}, J=8.3,7.1 \mathrm{~Hz}, 2 \mathrm{H}), 7.23-7.17$ $(\mathrm{m}, 3 \mathrm{H}), 5.93(\mathrm{dt}, J=5.8,2.4 \mathrm{~Hz}, 1 \mathrm{H}), 5.76(\mathrm{dt}, J=5.8,1.6 \mathrm{~Hz}, 1 \mathrm{H})$, 4.05-3.98 (m, $2 \mathrm{H}$ ), 3.97-3.89 (m, $2 \mathrm{H}$ ), 3.87 (dq, $J=7.7,2.0 \mathrm{~Hz}, 1 \mathrm{H}$ ), $2.77-2.66(\mathrm{~m}, 1 \mathrm{H}), 2.28(\mathrm{qd}, J=7.1,4.9 \mathrm{~Hz}, 1 \mathrm{H}), 1.86-1.81(\mathrm{~m}, 1 \mathrm{H})$, 1.78 (ddd, $J=14.1,5.0,1.7 \mathrm{~Hz}, 1 \mathrm{H}), 1.74-1.72(\mathrm{~m}, 1 \mathrm{H}), 1.69(\mathrm{dd}, J=$ $14.1,6.8 \mathrm{~Hz}, 1 \mathrm{H}), 1.63-1.57(\mathrm{~m}, 2 \mathrm{H})$.

${ }^{13} \mathrm{C}$ NMR $\left(151 \mathrm{MHz}, \mathrm{CDCl}_{3}\right): \delta=144.5,136.5,134.1,128.8,128.4$, 127.9, 126.7, 126.3, 109.5, 64.4, 64.1, 54.7, 48.7, 42.9, 34.2, 33.2, 26.5.

\section{(1R,2S)-2-Benzyl-2,3-dihydro-[1,1'-biphenyl]-4(1H)-one (8)}

Prepared from $\mathbf{4 c}$ via successive general procedures C, E, F followed by $C$ again. All intermediates were used crude without further purification. The intermediate after procedure $\mathrm{F}$ was purified via silica gel column chromatography (Hex/EtOAc 10\%) but not characterized $(27 \mathrm{mg}$, $62 \%$ over 3 steps). Final compound 8 was obtained after general procedure C [colorless oil, $27 \mathrm{mg}$, quant. (or $62 \%$ over 4 steps), 9:1 dr, major reported]. Solvent system for chromatography: Hex/EtOAc $20 \%$.

${ }^{1} \mathrm{H}$ NMR $\left(600 \mathrm{MHz}, \mathrm{CDCl}_{3}\right): \delta=7.43-7.31(\mathrm{~m}, 3 \mathrm{H}), 7.24(\mathrm{tt}, J=6.1,1.6$ $\mathrm{Hz}, 4 \mathrm{H}), 7.22-7.16(\mathrm{~m}, 1 \mathrm{H}), 7.02-6.98(\mathrm{~m}, 2 \mathrm{H}), 6.86$ (dd, $J=10.1,2.7$ $\mathrm{Hz}, 1 \mathrm{H}$ ), 6.13 (ddd, $J=10.0,2.7,0.8 \mathrm{~Hz}, 1 \mathrm{H}), 3.46(\mathrm{dt}, J=8.6,2.6 \mathrm{~Hz}, 1$ $\mathrm{H}), 2.78(\mathrm{dd}, J=12.8,3.5 \mathrm{~Hz}, 1 \mathrm{H}), 2.52(\mathrm{ddd}, J=16.2,3.7,0.9 \mathrm{~Hz}, 1 \mathrm{H}$ ), 2.49-2.43 (m, $1 \mathrm{H}), 2.43-2.39$ (m, $1 \mathrm{H}), 2.21$ (dd, $J=16.2,11.2 \mathrm{~Hz}, 1 \mathrm{H}$ ). ${ }^{13} \mathrm{C}$ NMR $\left(151 \mathrm{MHz}, \mathrm{CDCl}_{3}\right): \delta=199.5,152.8,141.8,139.0,129.4$, 129.2, 129.1, 128.6, 128.5, 127.5, 126.5, 48.8, 44.8, 42.1, 39.9.

HRMS (ESI-TOF): $m / z$ [M $+\mathrm{H}]^{+}$calcd for $\mathrm{C}_{19} \mathrm{H}_{19} \mathrm{O}: 263.1430$; found: 263.1438 .

((1R,6S)-6-Benzyl-4-oxocyclohex-2-en-1-yl)methyl Acetate (9)

Prepared from $\mathbf{4 d}$ via successive general procedures C, E, F followed by $C$ again. All intermediates were used crude without further purification. The intermediate after procedure $\mathrm{F}$ was purified via silica gel column chromatography (Hex/EtOAc 10\%) but not characterized (9 mg, $52 \%$ over 3 steps). Final compound 9 was obtained after general procedure C [colorless oil, $9 \mathrm{mg}$, quant. (or $52 \%$ over 4 steps), 2:1 dr]. Solvent system for chromatography: Hex/EtOAc 30\%.

${ }^{1} \mathrm{H} \mathrm{NMR}\left(600 \mathrm{MHz}, \mathrm{CDCl}_{3}\right): \delta=7.33-7.27(\mathrm{~m}, 2 \mathrm{H}), 7.25-7.19(\mathrm{~m}, 1 \mathrm{H})$, 7.17-7.10 (m, $2 \mathrm{H}), 6.88$ (dd, $J=10.1,4.6 \mathrm{~Hz}, 1 \mathrm{H}), 6.12$ (dd, $J=10.1$, $1.9 \mathrm{~Hz}, 1 \mathrm{H}), 4.39$ (dd, $J=11.2,5.8 \mathrm{~Hz}, 1 \mathrm{H}), 4.27(\mathrm{dd}, J=11.2,6.7 \mathrm{~Hz}, 1$ H), 2.85 (ddt, $J=7.3,4.2,2.3 \mathrm{~Hz}, 1 \mathrm{H}$ ), 2.81-2.77 (m, $1 \mathrm{H}$ ), 2.66-2.57 (m, $2 \mathrm{H}), 2.44-2.33(\mathrm{~m}, 2 \mathrm{H}), 2.08(\mathrm{~s}, 3 \mathrm{H})$.

${ }^{13} \mathrm{C}$ NMR $\left(151 \mathrm{MHz}, \mathrm{CDCl}_{3}\right): \delta=198.9,170.9,149.2,138.9,131.0$, 129.1, 128.8, 126.7, 63.2, 40.8, 38.5, 38.3, 37.9, 21.1.

HRMS (ESI-TOF): $m / z\left[\mathrm{M}+\mathrm{NH}_{4}\right]^{+}$calcd for $\mathrm{C}_{16} \mathrm{H}_{22} \mathrm{NO}_{3}: 276.1594$; found: 276.1601 .

\section{Funding Information}

This material is based upon work supported by the National Science Foundation under Grant No. 1844443. We thank the College of Liberal Arts and Sciences and the Department of Chemistry at the University of Florida for start-up funds. We thank the Mass Spectrometry Research and Education Center and their funding source: NIH S10 OD021758-01A1.

\section{Supporting Information}

Supporting information for this article is available online at https://doi.org/10.1055/s-0040-1707184. Additional experimental details and spectroscopic reprints (1H and 13C NMR) are available.

\section{References}

(1) Freeman, F. Chem. Rev. 1980, 80, 329.

(2) Li, X. M.; Wang, B.; Zhang, J. M.; Yan, M. Org. Lett. 2011, 13, 374.

(3) Hu, Z. P.; Lou, C. L.; Wang, J. J.; Chen, C. X.; Yan, M. J. Org. Chem. 2011, 76, 3797.

(4) Molleti, N.; Rana, N. K.; Singh, V. K. Org. Lett. 2012, 14, 4322.

(5) Jing, L.; Wei, J.; Zhou, L.; Huang, Z.; Li, Z.; Wu, D.; Xiang, H.; Zhou, X. Chem. Eur. J. 2010, 16, 10955.

(6) Tayyari, F.; Wood, D. E.; Fanwick, P. E.; Sammelson, R. E. Synthesis 2008, 279.

(7) Sammelson, R. E.; Allen, M. J. Synthesis 2005, 543.

(8) Förster, S.; Tverskoy, O.; Helmchen, G. Synlett 2008, 2803.

(9) Amundsen, L. H.; Nelson, L. S. J. Am. Chem. Soc. 1951, 73, 242.

(10) Haddenham, D.; Pasumansky, L.; DeSoto, J.; Eagon, S.; Singaram, B. J. Org. Chem. 2009, 74, 1964.

(11) Mattalia, J.-M. R. Beilstein J. Org. Chem. 2017, 13, 267.

(12) Mattalia, J.-M.; Marchi-Delapierre, C.; Hazimeh, H.; Chanon, M. ARKIVOC 2006, (iv), 90.

(13) Rao, J. A.; Ravichandran, K.; Malley, G. J. O.; Cava, M. P. Can. J. Chem. 1987, 65, 31.

(14) Watt, D. S. J. Org. Chem. 1974, 39, 2799.

(15) Freerksen, R. W.; Selikson, S. J.; Wroble, R. R.; Kyler, K. S.; Watt, D. S. J. Org. Chem. 1983, 48, 4087.

(16) DiBiase, S. A.; Wolak, R. P.; Dishong, D. M.; Gokel, G. W. J. Org. Chem. 1980, 45, 3630.

(17) Kulp, S. S.; McGee, M. J. J. Org. Chem. 1983, 48, 4097.

(18) Selikson, S. J.J. Org. Chem. 1975, 40, 267.

(19) Li, J.; Lear, M. J.; Hayashi, Y. Angew. Chem. Int. Ed. 2016, 55, 9060.

(20) Hayashi, Y.; Li, J.; Asano, H.; Sakamoto, D. Eur. J. Org. Chem. 2019, 675 .

(21) Lahtigui, O.; Emmetiere, F.; Zhang, W.; Jirmo, L.; Toledo-Roy, S.; Hershberger, J. C.; Macho, J. M.; Grenning, A. J. Angew. Chem. Int. Ed. 2016, 55, 15792.

(22) Ullrich, V. Angew. Chem., Int. Ed. Engl. 1972, 11, 701.

(23) Fréchet, J. M. J. Tetrahedron 1981, 37, 663.

(24) Mimoun, H. J. Mol. Catal. 1980, 7, 1.

(25) Omura, T.; Sato, R. J. Biol. Chem. 1964, 239, 2370.

(26) Iwao, T.; Noboru, K. J. Am. Chem. Soc. 1979, 101, 6456.

(27) Klingenberg, M. Arch. Biochem. Biophys. 1958, 75, 376.

(28) Shimizu, M.; Orita, H.; Hayakawa, T.; Takehira, K. J. Mol. Catal. 1988, $45,85$.

(29) Shimizu, M.; Orita, H.; Hayakawa, T.; Takehira, K. J. Mol. Catal. 1989, 53, 165.

(30) Nishiki, M.; Satoh, T.; Sakurai, H. J. Mol. Catal. 1990, 62, 79.

(31) Perrée-Fauvet, M.; Gaudemer, A. J. Chem. Soc., Chem. Commun. 1981, 874.

(32) Takeuchi, M.; Kodera, M.; Kano, K.; Yoshida, Z. I. J. Mol. Catal. A: Chem. 1996, 113, 51.

(33) Hill, C. L.; Whitesides, G. M. J. Am. Chem. Soc. 1974, 96, 870.

(34) Isayama, S.; Mukaiyama, T. Chem. Lett. 1989, 569.

(35) Isayama, S.; Mukaiyama, T. Chem. Lett. 1989, 573.

(36) Isayama, S. Bull. Chem. Soc. Jpn. 1990, 63, 1305.

(37) Inoki, S.; Kato, K.; Isayama, S.; Mukaiyama, T. Chem. Lett. 1990, 1869.

(38) Isayama, S.; Mukaiyama, T. Chem. Lett. 1989, 1071. 
(39) Crossley, S. W. M.; Obradors, C.; Martinez, R. M.; Shenvi, R. A. Chem. Rev. 2016, 116, 8912.

(40) Teichert, J. F.; den Hartog, T.; Hanstein, M.; Smit, C.; ter Horst, B.; Hernandez-Olmos, V.; Feringa, B. L.; Minnaard, A. J. ACS Catal. 2011, 1, 309.

(41) Ruider, S. A.; Sandmeier, T.; Carreira, E. M. Angew. Chem. Int. Ed. 2015, 54, 2378.

(42) Bosch, C.; Fiser, B.; Gómez-Bengoa, E.; Bradshaw, B.; Bonjoch, J. Org. Lett. 2015, 17, 5084.

(43) Iwasaki, K.; Wan, K. K.; Oppedisano, A.; Crossley, S. W. M.; Shenvi, R. A. J. Am. Chem. Soc. 2014, 136, 1300.

(44) Lu, H. H.; Pronin, S. V.; Antonova-Koch, Y.; Meister, S.; Winzeler, E. A.; Shenvi, R. A. J. Am. Chem. Soc. 2016, 138, 7268.

(45) Lackner, A. D.; Samant, A. V.; Toste, F. D. J. Am. Chem. Soc. 2013, $135,14090$.

(46) Andrez, J.-C.; Chowdhury, S.; Decker, S.; Dehnhardt, C. M.; Focken, T.; Grimwood, M. E.; Hemeon, I. W.; Jia, Q.; Li, J.; Ortwine, D. F.; Safina, B.; Sheng, T.; Sun, S.; Sutherlin, D. P.; Wilson, M. S.; Zenova, A. Y. WO 2013177224 A1, 2013.

(47) Zhang, K.; Chang, L.; An, Q.; Wang, X.; Zuo, Z. J. Am. Chem. Soc. 2019, 141, 10556.

(48) Scott, S. K.; Grenning, A. J. Angew. Chem. Int. Ed. 2017, 56, 8125.

(49) Scott, S. K.; Sanders, J. N.; White, K. E.; Yu, R. A.; Houk, K. N.; Grenning, A. J.J. Am. Chem. Soc. 2018, 140, 16134.

(50) Fereyduni, E.; Grenning, A. J. Org. Lett. 2017, 19, 4130.

(51) Fereyduni, E.; Sanders, J. N.; Gonzalez, G.; Houk, K. N.; Grenning, A. J. Chem. Sci. 2018, 9, 8760.
(52) Schroeder, R.; Grenning, A. J. Tetrahedron 2019, 75, 3231.

(53) Vertesaljai, P.; Serrano, R.; Mannchen, M. D.; Williams, M.; Semenova, E.; Grenning, A. J. Org. Lett. 2019, 21, 5704.

(54) Ilardi, E. A.; Stivala, C. E.; Zakarian, A. Chem. Soc. Rev. 2009, 38 , 3133.

(55) Jones, A. C.; May, J. A.; Sarpong, R.; Stoltz, B. M. Angew. Chem. Int. Ed. 2014, 53, 2556.

(56) Cosgrove, K. L.; McGeary, R. P. Tetrahedron 2010, 66, 3050.

(57) Sun, Z.; Li, Z.; Liao, W. W. Green Chem. 2019, 21, 1614.

(58) Manojveer, S.; Salahi, S.; Wendt, O. F.; Johnson, M. T. J. Org. Chem. 2018, 83, 10864.

(59) Chen, Y.; Leonardi, M.; Dingwall, P.; Labes, R.; Pasau, P.; Blakemore, D. C.; Ley, S. V.J. Org. Chem. 2018, 83, 15558.

(60) Taylor, S. K.; Dickinson, M. G.; May, S. A.; Pickering, D. A.; Sadek, P. C. Synthesis 1998, 1133.

(61) Akhtar, W. M.; Armstrong, R. J.; Frost, J. R.; Stevenson, N. G.; Donohoe, T. J.J. Am. Chem. Soc. 2018, 140, 11916.

(62) Dolente, C.; Schnider, P. US 20110275801 A1, 2011

(63) Cueva, J. P.; Gallardo-Godoy, A.; Juncosa, J. I.; Vidi, P. A.; Lill, M. A.; Watts, V. J.; Nichols, D. E. J. Med. Chem. 2011, 54, 5508.

(64) Li, Z.; Gupta, M. K.; Snowden, T. S. Eur. J. Org. Chem. 2015, 7009.

(65) Ingerl, A.; Justus, K.; Hellwig, V.; Steglich, W. Tetrahedron 2007, $63,6548$.

(66) van Greunen, D. G.; Cordier, W.; Nell, M.; van der Westhuyzen, C.; Steenkamp, V.; Panayides, J. L.; Riley, D. L. Eur. J. Med. Chem. 2017, 127, 671. 